\title{
A CONGRUENCE-FREE INVERSE SEMIGROUP ASSOCIATED WITH A PAIR OF INFINITE CARDINALS
}

\author{
J. M. HOWIE \\ (Received 18 October 1980) \\ Communicated by T. E. Hall
}

\begin{abstract}
Let $m, n$ be infinite cardinals such that $m>n$, and let $X$ be a set of cardinality $m$. Within the symmetric inverse semigroup on $X$ the elements whose domain and range have complements of cardinality $m$ form an inverse semigroup $T$. The closure $E \omega$ of the semilattice $E$ of idempotents of $T$ is a fundamental bisimple inverse semigroup. Its maximum congruence is described. The quotient of $E \omega$ by this maximum congruence is a bisimple, congruence-free inverse semigroup.
\end{abstract}

1980 Mathematics subject classification (Amer. Math. Soc.): 20 M 20.

\section{Introduction}

In a previous paper (1981) a regular, bisimple, idempotent-generated, congruence-free semigroup was found to be associated with each infinite regular cardinal. The main result of this paper is not in any strict sense analogous to the earlier result, though the methods do have something in common. In particular, the invocation at a crucial stage in (Howie 1981) of Mal'cev's (1952) description of the congruences on a full transformation semigroup is closely paralleled in this paper by the quoting of the analogous description due to Liber (1953) of the congruences on a symmetric inverse semigroup. There is some connection too with the ideas in (Štov 1960, 1963).

Proving that a semigroup is congruence-free seems rarely to be easy. The method used here owes much to the study of congruence-free inverse semigroups in (Munn 1974, 1975).

Copyright Australian Mathematical Society 1981 
For basic notation and terminology in semigroup theory see (Howie 1976).

\section{Various inverse semigroups associated with a pair of infinite cardinals}

Let $\mathbf{m}, \mathbf{n}$ be infinite cardinals, with $\mathbf{m}>\mathbf{n}$, and let $X$ be a set of cardinality $\mathbf{m}$. Let $g(X)$ be the symmetric inverse semigroup on the set $X$ and let

$$
T=T_{\mathbf{m}, \mathbf{m}}=\{\alpha \in \mathcal{G}(X):|X \backslash \operatorname{dom} \alpha|=|X \backslash \operatorname{ran} \alpha|=\mathbf{n}\} .
$$

Then

LEMMA 1. $T$ is an inverse subsemigroup of $9(X)$.

Proof. Let $\alpha, \beta \in T$. If $x \in X \backslash \operatorname{dom} \alpha \beta$ then either $x \in X \backslash \operatorname{dom} \alpha$ or $x \in(\operatorname{ran} \alpha \backslash \operatorname{dom} \beta) \alpha^{-1}$. Thus

$$
|X \backslash \operatorname{dom} \alpha \beta| \leqslant|X \backslash \operatorname{dom} \alpha|+|X \backslash \operatorname{dom} \beta|=\mathbf{n} .
$$

Since we also have

$$
X \backslash \operatorname{dom} \alpha \beta \supseteq X \backslash \operatorname{dom} \alpha,
$$

it follows that $|X \backslash \operatorname{dom} \alpha \beta|=\mathbf{n}$. Similarly, if $x \in X \backslash \operatorname{ran} \alpha \beta$ then either $x \in$ $X \backslash \operatorname{ran} \beta$ or $x \in(\operatorname{dom} \beta \backslash \operatorname{ran} \alpha) \beta$. Hence

$$
|X \backslash \operatorname{ran} \alpha \beta| \leqslant|X \backslash \operatorname{ran} \beta|+|X \backslash \operatorname{ran} \alpha|=\mathbf{n} .
$$

Since we also have

$$
X \backslash \operatorname{ran} \alpha \beta \supseteq X \backslash \operatorname{ran} \beta,
$$

it follows that $|X \backslash \operatorname{ran} \alpha \beta|=\mathbf{n}$. Thus $\alpha \beta \in T$.

Since $\operatorname{dom} \alpha^{-1}=\operatorname{ran} \alpha$ and $\operatorname{ran} \alpha^{-1}=\operatorname{dom} \alpha$ it is clear that

$$
\alpha \in T \Rightarrow \alpha^{-1} \in T
$$

thus $T$ is an inverse subsemigroup of $\mathscr{G}(X)$.

The set $E$ of idempotents of $T$ is given by

$$
E=\left\{1_{A}: A \in C_{\mathrm{n}}(X)\right\},
$$

where $C_{\mathrm{n}}(X)$ is the set of all subsets $Y$ of $X$ for which $|X \backslash Y|=\mathbf{n}$. We now describe the closure

$$
E \omega=\{\alpha \in T:(\exists \varepsilon \in E) \varepsilon<\alpha\}
$$

of $E$ in $T$. For $\alpha$ in $T$, define $S(\alpha)$, the shift of $\alpha$, to be $\{x \in X: x \alpha \neq x\}$. Then

LEMMA 2. $E \omega=\{\alpha \in T:|S(\alpha)|<\mathbf{n}\}$. 
Proof. Suppose first that $\alpha \in E \omega$. Then there exists $1_{A} \in E$ such that $1_{A}<\alpha$. Thus $A \subseteq \operatorname{dom} \alpha$ and $\alpha \mid A=1_{A}$. Now $|X \backslash A|=\mathbf{n}$ and so, since $S(\alpha) \subseteq$ $\operatorname{dom} \alpha \backslash A$, we have

$$
|S(\alpha)|<|X \backslash A|=\mathbf{n} .
$$

Suppose now that $|S(\alpha)|<\mathbf{n}$. Choose a subset $D$ of $X$ such that

$$
S(\alpha) \subseteq D \subset \operatorname{dom} \alpha, \quad|D|=\mathbf{n}
$$

this is possible since $|\operatorname{dom} \alpha|=\mathbf{m}>\mathbf{n}$. Let $A=\operatorname{dom} \alpha \backslash D$; then $|X \backslash A|=\mathbf{n}$, since

$$
X \backslash A=(X \backslash \operatorname{dom} \alpha) \cup D .
$$

Thus $1_{A} \in E$, and since $\alpha \mid A=1_{A}$ we have $1_{A}<\alpha$. Thus $\alpha \in E \omega$.

Let us write $E \omega=U$. Then $U$ is an inverse semigroup and has the following interesting properties:

LEMMA 3. $U$ is fundamental and has the property that its only group homomorphic image is trivial.

Proof. The second property is obvious since $U=E \omega$. To show that $U$ is fundamental it is sufficient to show that $E \zeta=E$, where

$$
E \zeta=\{\alpha \in U:(\forall \varepsilon \in E) \alpha \varepsilon=\varepsilon \alpha\}
$$

is the centraliser of $E$ in $U$. (See (Howie 1964).) It is clear that $E \zeta \supseteq E$; we complete the proof by showing that if $\alpha \notin E$ then $\alpha \notin E \zeta$. For if $\alpha \in U \backslash E$ then there exists $x$ in dom $\alpha$ such that $x \alpha \neq x$. Choose a subset $B$ in $C_{\mathrm{n}}(X)$ such that $x \in B, x \alpha \notin B$. Then $x \in \operatorname{dom}\left(1_{B} \alpha\right), x \notin \operatorname{dom}\left(\alpha 1_{B}\right)$ and so $1_{B} \alpha \neq \alpha 1_{B}$.

A further interesting property of $U$ is given by

LEMMA 4. $U$ is bisimple.

Proof. Let $1_{A}, 1_{B} \in E$. It will be sufficient to show that $\left(1_{A}, 1_{B}\right) \in \mathscr{Q}$. Since

$$
X \backslash(A \cap B)=(X \backslash A) \cup(X \backslash B),
$$

it follows that $|X \backslash(A \cap B)|=\mathbf{n}$. Hence $|A \cap B|=\mathbf{m}$ and we may partition $A \cap B$ into disjoint subsets $C, D$ with $|C|=\mathrm{m},|D|=\mathbf{n}$. Since

$$
|A \backslash B|<|X \backslash B|=\mathbf{n} \quad \text { and } \quad|B \backslash A|<|X \backslash A|=\mathbf{n},
$$

it follows that

$$
|(A \backslash B) \cup D|=|(B \backslash A) \cup D|=\mathbf{n} .
$$


Let $\theta:(A \backslash B) \cup D \rightarrow(B \backslash A) \cup D$ be a bijection and let $\alpha=\theta \cup 1_{C}$. Then $\operatorname{dom} \alpha=A, \operatorname{ran} \alpha=B$ and

$$
|S(\alpha)| \leqslant|(A \backslash B) \cup D|=\mathbf{n} .
$$

So $\alpha \in U$ and $\alpha \Re 1_{A}, \alpha \mathcal{L} 1_{B}$. Thus $\left(1_{A}, 1_{B}\right) \in \mathscr{D}$ and so $U$ is bisimple.

Since $U$ has no non-trivial group congruence and no non-trivial idempotent separating congruence one might conjecture that it is congruence-free. However, it follows from the work of Liber (1953) that for each infinite $\mathbf{p}<\mathbf{n}$ the relation

$$
\delta_{\mathbf{p}}=\{(\alpha, \beta) \in U \times U:|(\alpha \backslash \beta) \cup(\beta \backslash \alpha)|<\mathbf{p}\}
$$

is a congruence on $U$. Of these congruences the greatest is $\delta_{\mathbf{n}}$ and it is reasonable to ask whether the inverse semigroup $U^{*}=U / \delta_{\mathrm{n}}$ is congruence-free.

Some of the properties of $U^{*}$ we know already. It is bisimple and its only group homomorphic image is trivial-these being properties it inherits as a homomorphic image of $U$. It also inherits the property of being fundamental, but this is less obvious, and before we can show it we require a preliminary lemma:

LEMMA 5. If $\alpha \in U$ is such that $|S(\alpha)|=\mathbf{n}$ then there exists a subset $Z$ of $S(\alpha)$ such that $|Z|=\mathbf{n}$ and $Z \alpha \cap Z=\varnothing$.

Proof. We apply Zorn's Lemma to the set $\mathcal{Y}$ of subsets $Y$ of $S(\alpha)$ such that $Y \alpha \cap Y=\varnothing$. Certainly $\mathcal{Y} \neq \varnothing$, since $\{y\} \alpha \cap\{y\}=\varnothing$ for all $y$ in $S(\alpha)$. If $\left(Y_{\lambda}\right)$ is a chain in $(\mathcal{Y}, \subseteq)$ then $Y=\cup Y_{\lambda} \in \mathcal{Y}$, since if $y \in Y \cap Y \alpha$ then $y \in Y_{\lambda}$ and $y \in Y_{\mu} \alpha$ for some $\lambda, \mu$; thus $y \in Y_{\nu} \cap Y_{\nu} \alpha$, where $\nu=\max (\lambda, \mu)$, which is a contradiction.

Let $Z$ be a maximal element of $\mathcal{Y}$. We show that $|Z|=\mathbf{n}$. For suppose by way of contradiction that $|Z|<\mathbf{n}$. Then $|Z \alpha|=|Z|<\mathbf{n}$ also and so $|S(\alpha) \backslash(Z \cup Z \alpha)|=\mathbf{n}$. Let $t \in S(\alpha) \backslash(Z \cup Z \alpha)$. If $t \alpha \notin Z$ then (since certainly $t \alpha \neq t$ ) the set $Z \cup\{t\} \in \mathcal{Y}$. This is impossible since $Z$ is maximal; hence $t \alpha \in Z$. But then the one-one mapping $\alpha$ maps $S(\alpha) \backslash(Z \cup Z \alpha)$ (of cardinality n) into $Z$ (of cardinality less than $\mathbf{n}$ ). This being impossible, we conclude that $|Z|=\mathbf{n}$.

We can now prove

LEMMA 6. $U^{*}$ is fundamental.

Proof. Again we show that $E^{*} \zeta=E^{*}$, where $E^{*}=E \delta_{n}^{\natural}$ is the semilattice of idempotents of $U^{*}$. Suppose that $\alpha \delta_{\mathbf{n}} \notin E^{*}$, where $\alpha \in U$. If $|S(\alpha)|<\mathbf{n}$ then $\left(\alpha, 1_{Y}\right) \in \delta_{m}$, where $Y=\{x: x \alpha=x\} \in C_{n}(X)$, and so $\alpha \delta_{n} \in E^{*} ;$ hence we 
may suppose that $|S(\alpha)|=\mathbf{n}$. By Lemma 5 there exists $Z \subseteq S(\alpha)$ such that $|Z|=\mathbf{n}$ and $Z \alpha \cap Z=\varnothing$. Hence for all $z$ in $Z$ we have $z \in \operatorname{dom} 1_{Z} \alpha$ while $z \notin \operatorname{dom} \alpha 1_{z}$. Thus $\left(1_{z} \alpha, \alpha 1_{z}\right) \notin \delta_{\mathrm{n}}$ and so

$$
\left(\alpha \delta_{\mathrm{n}}\right)\left(1_{Z} \delta_{\mathrm{n}}\right) \neq\left(1_{Z} \delta_{\mathrm{n}}\right)\left(\alpha \delta_{\mathrm{n}}\right)
$$

in $U^{*}$. We have shown that if $\alpha \delta_{\mathrm{n}} \notin E^{*}$ then $\alpha \delta_{\mathrm{n}} \notin E^{*} \zeta$. Hence $E^{*} \zeta=E^{*}$ as required, and $U^{*}$ is fundamental.

Having shown that $U^{*}$ has no non-universal group congruence and no non-trivial idempotent separating congruence, we now invoke the following result due to Munn (1974):

LEMMA 7. Let $S$ be an inverse semigroup. Then $S$ has the property that every congruence is either idempotent-separating or a group congruence if and only if it has the following property:

(C) Let $a, b, c, d$ be idempotents of $S$ such that $a>b, c>d$. Then for some $n>1$ there exist $z_{1}, \ldots, z_{n}$ in $S$ such that

$$
z_{1}^{-1} u_{1} z_{1}=c, z_{1}^{-1} v_{1} z_{1}=z_{2}^{-1} u_{2} z_{2}, \ldots, z_{n-1}^{-1} v_{n-1} z_{n-1}=z_{n}^{-1} u_{n} z_{n}, z_{n}^{-1} v_{n} z_{n}=d,
$$

where, for each $i,\left(u_{i}, v_{i}\right)$ is either $(a, b)$ or $(b, a)$.

We show that $U^{*}$ satisfies the condition (C). Let $1_{A}, 1_{B}, 1_{C}, 1_{D}$ be idempotents in $U$ such that $1_{A} \delta_{\mathrm{n}}>1_{B} \delta_{\mathrm{n}}, 1_{C} \delta_{\mathrm{n}}>1_{D} \delta_{\mathrm{n}}$ in $U^{*}$. Then $\left(1_{A \cap B}, 1_{B}\right)=\left(1_{A} 1_{B}, 1_{B}\right)$ $\in \delta_{\mathbf{n}}$ and so

$$
1_{B} \delta_{\mathrm{n}}=1_{A \cap B} \delta_{\mathrm{n}}
$$

Also, since $\left(1_{A}, 1_{A \cap B}\right) \notin \delta_{\mathrm{n}}$ we have $|A \backslash(A \cap B)| \geqslant \mathrm{n}$. In fact

$$
|A \backslash(A \cap B)|=\mathbf{n},
$$

since $A \backslash(A \cap B) \subseteq X \backslash B$ and $B \in C_{\mathrm{n}}(X)$. Similarly,

$$
1_{C} \delta_{\mathbf{n}}=1_{C \cap D} \delta_{\mathbf{n}},|C \backslash(C \cap D)|=\mathbf{n} \text {. }
$$

Now let $\theta: A \backslash(A \cap B) \rightarrow C \backslash(C \cap D)$ be a bijection. Since

$$
\begin{gathered}
|A \cap B|=|C \cap D|=|A \cap B \cap C \cap D|=\mathbf{m}, \\
|(A \cap B) \backslash(C \cap D)| \leqslant \mathbf{n} \text { and }|(C \cap D) \backslash(A \cap B)| \leqslant \mathbf{n},
\end{gathered}
$$

it is possible to choose a bijection $\varphi: A \cap B \rightarrow C \cap D$ such that $S(\varphi) \leqslant \mathbf{n}$. Let $\zeta_{1}=\theta \cup \varphi$. Then

$$
S\left(\zeta_{1}\right) \subseteq(A \backslash(A \cap B)) \cup S(\varphi)
$$

and so $\left|S\left(\zeta_{1}\right)\right| \leqslant \mathbf{n}$. Since $\operatorname{dom} \zeta_{1}=A$ and $\operatorname{ran} \zeta_{1}=C$ we have $\zeta_{1} \in U$. Moreover,

$$
\zeta_{1}^{-1} 1_{A} \zeta_{1}=1_{C}, \quad \zeta_{1}^{-1} 1_{A \cap B} \zeta_{1}=1_{C \cap D}
$$


and so in $U^{*}$

$$
\left(\zeta_{1} \delta_{\mathrm{n}}\right)^{-1}\left(1_{A} \delta_{\mathrm{n}}\right)\left(\zeta_{1} \delta_{\mathrm{n}}\right)=1_{C} \delta_{\mathrm{n}}, \quad\left(\zeta_{1} \delta_{\mathrm{n}}\right)^{-1}\left(1_{B} \delta_{\mathrm{n}}\right)\left(\zeta_{1} \delta_{\mathrm{n}}\right)=1_{D} \delta_{\mathrm{n}}
$$

Thus $U^{*}$ satisfies the criterion (C) with $n=1$.

It follows that $U^{*}$ is congruence-free, since we now know that it has no non-universal group congruence, no non-trivial idempotent-separating congruence, and no congruence that is not either a group congruence or idempotent-separating. We summarise our results in a theorem as follows:

THEOREM. Let $\mathbf{m}, \mathbf{n}$ be infinite cardinals such that $\mathbf{m}>\mathbf{n}$. Let $X$ be a set of cardinality $\mathbf{m}$ and let

$$
U=\{\alpha \in G(X):|X \backslash \operatorname{dom} \alpha|=|X \backslash \operatorname{ran} \alpha|=\mathbf{n},|S(\alpha)|<\mathbf{n}\} .
$$

Let $U^{*}=U / \delta_{n}$, where $\delta_{\mathrm{n}}$ is the congruence on $U$ given by

$$
\delta_{\mathbf{n}}=\{(\alpha, \beta) \in U \times U:|(\alpha \backslash \beta) \cup(\beta \backslash \alpha)|<\mathbf{n}\} .
$$

Then $U^{*}$ is a bisimple congruence-free inverse semigroup.

\section{References}

J. M. Howie (1964), 'The maximum idempotent-separating congruence on an inverse semigroup', Proc. Edinburgh Math. Soc. (2) 14, 71-79.

J. M. Howie (1976), An introduction to semigroup theory (Academic Press, London).

J. M. Howie (1981), 'A class of bisimple idempotent-generated congruence-free semigroups', Proc. Roy. Soc. Edinburgh A 88A, 169-184.

A. E. Liber (1953), 'On symmetric generalised groups', Mat. Sb. 33 (75), 531-544 (in Russian).

W. D. Munn (1974), 'Congruence-free inverse semigroups', Quart. J. Math. Oxford (Ser.) 25, 463-484.

W. D. Munn (1975), 'A note on congruence-free semigroups', Quart. J. Math. Oxford (Ser.) 26, 385-387.

E. G. Sutov (1960), 'On semigroups of almost identical transformations', Dakl. Akad. Nauk SSSR 134, 292-295 (in Russian).

E. G. Sutov (1963), 'Homomorphisms of the semigroup of all near-identity mappings', Izv. Vyss. Učebn. Zaved. Matematika no. 2 (33), 176-180.

Mathematical Institute University of St. Andrews

Scotland 